\title{
Magnetic resonance imaging of dilated cardiomyopathy: prognostic benefit of identifying late gadolinium enhancement in Asian patients
}

\author{
Anna Nogue Infante ${ }^{1}$, Bsc, Christopher Chieh Yang $\underline{K o O}^{2}$, мBвs, Alfred Yip ${ }^{2}$, мBвs, Ying Ha $\underline{\mathrm{Lim}}^{2}$, мBBs, \\ Wee Tiong $\mathrm{YeO}^{2}$, MBBS, Swee Tian Quek ${ }^{3}$, MBBs, Toon Wei Lim², MBBS, PhD, Swee Chong Seow ${ }^{2}$, MBBS, \\ Ping $\underline{\text { Chai }}^{2}$, MBBS, Ching Ching $\underline{\text { Ong }}^{3}$, MBBs, Lynette $\underline{\text { Teo }}^{3}$, MBBs, Devinder $\underline{\text { Singh }}^{2}$, MBBs, Pipin Kojodjojo ${ }^{2}$, MBBS, PhD
}

INTRODUCTION Risk stratification in dilated cardiomyopathy (DCM) is imprecise, relying largely on echocardiographic left ventricular ejection fraction (LVEF) and severity of heart failure symptoms. Adverse cardiovascular events are increased by the presence of myocardial scarring. Late gadolinium enhancement (LGE) on cardiovascular magnetic resonance (CMR) imaging is the gold standard for identifying myocardial scars. We examined the association between LGE on CMR imaging and adverse clinical outcomes during long-term follow-up of Asian patients with DCM.

METHODS Consecutive patients with DCM undergoing CMR imaging at a single Asian academic medical centre between 2005 and 2015 were recruited. Clinical outcomes were tracked using comprehensive electronic medical records and mortality was determined by cross-linkages with national registries. Presence and distribution of LGE on CMR imaging were determined by investigators blinded to patient outcomes. Primary endpoint was a composite of heart failure hospitalisations, appropriate implantable cardioverter-defibrillator shocks and cardiovascular mortality.

RESULTS Of 86 patients, $64.0 \%$ had LGE (80.2\% male; mean LVEF $30.1 \% \pm 12.7 \%)$. Mid-wall fibrosis (71.7\%) was the most common pattern of LGE distribution. Over a mean follow-up period of $4.9 \pm 3.2$ years, $19(34.5 \%)$ patients with LGE reached the composite endpoint compared to $4(12.9 \%)$ patients without LGE $(p=0.01)$. Presence of LGE, but not echocardiographic LVEF, independently predicted the primary endpoint (hazard ratio 4.15 [95\% confidence interval 1.28-13.50]; $p=0.02$ ). CONCLUSION LGE presence independently predicted adverse clinical events in Asian patients with DCM. Routine use of CMR imaging to characterise the myocardial substrate is recommended for enhanced risk stratification and should strongly influence clinical management.

Keywords: dilated cardiomyopathy, magnetic resonance imaging, prognosis

\section{INTRODUCTION}

Risk stratification in non-ischaemic dilated cardiomyopathy (DCM) is imprecise, with current guidelines focusing on left ventricular ejection fraction (LVEF) and heart failure symptom status. ${ }^{(1-3)}$ Markers for increased risk of sudden death, validated in ischaemic cardiomyopathy, perform less well for DCM. ${ }^{(4)}$ Consequently, solely relying upon these parameters to risk stratify patients with DCM lowers the clinical efficacy and cost-effectiveness of primary prevention implantable cardioverter-defibrillator (ICD). On the other hand, at-risk patients with LVEF above the recommended cut-off may be excluded from ICD implantation.

A majority of sudden cardiac deaths are caused by ventricular arrhythmias, which in turn arise from re-entrant activity around heterogeneous myocardial scars. ${ }^{(5,6)}$ Cardiovascular magnetic resonance $(\mathrm{CMR})$ imaging is established as the gold standard for quantification and accurate identification of myocardial scarring by the detection of late gadolinium enhancement (LGE). Western cohorts of patients with DCM with LGE on CMR imaging have higher risks of adverse cardiac events, regardless of LVEF and heart failure symptom status. ${ }^{(7-10)}$ In this study, we examined the characteristics of myocardial scarring in consecutive Asian patients with DCM and their association with long-term adverse clinical outcomes.

\section{METHODS}

The study population was extracted from a prospective registry of consecutive patients attending the National University Hospital, Singapore, for clinically indicated gadolinium-enhanced CMR imaging between 2005 and 2015. Patients were included if they had: (a) LVEF $\leq 50 \%$ on transthoracic echocardiography; (b) absence of significant obstructive stenosis on coronary angiography, normal stress echocardiography or myocardial perfusion scanning to exclude ischaemic heart disease; and (c) symptoms exceeding six weeks in duration before undergoing CMR imaging to avoid inclusion of patients with acute myocarditis.

Exclusion criteria were: (a) specific diagnosis of hypertrophic cardiomyopathy, sarcoidosis, arrhythmogenic right ventricular dysplasia and infiltrative cardiac disease, such as amyloidosis and cardiac lymphoma; and (b) patients who were not permanent residents or citizens of Singapore, as their mortality and cause of death could not be tracked by cross-linkage with national registries.

CMR imaging was performed using a 1.5T scanner (MAGNETOM Symphony or MAGNETOM Aera; Siemens Healthcare, Erlangen, Bavaria, Germany). Steady-state free precession breath-hold sequences (TE[echo time]/TR[repetition

${ }^{1}$ Department of Biomedical Engineering, Universitat Pompeu Fabra, Barcelona, Spain, ${ }^{2}$ Department of Cardiology, National University Heart Centre Singapore, ${ }^{3}$ Department of Diagnostic Imaging, National University Hospital, Singapore

Correspondence: Dr Pipin Kojodjojo, Senior Consultant, Department of Cardiology, National University Heart Centre Singapore, 1E Kent Ridge Road, NUHS Tower Block, Level 9, Singapore 119228. pipin_kojodjojo@nuhs.edu.sg 
time $=1.1 / 40 \mathrm{msec}, 25$ phases per cardiac cycle, matrix size $192 \times 192$ ) were used to acquire cine images in standard longaxis planes (slice thickness $8 \mathrm{~mm}$ ) and in sequential $8-\mathrm{mm}$ short-axis slices (with 2-mm gap) from the atrioventricular ring to the ventricular apex. LGE magnetic resonance imaging for the characterisation of myocardial scar was obtained as twodimensional phase-sensitive inversion recovery sequences in identical planes to the cine images 10-15 minutes after the administration of intravenous gadolinium contrast (gadopentetate dimeglumine $0.2 \mathrm{mg} / \mathrm{kg}$ or gadobutrol $0.1 \mathrm{~mL} / \mathrm{kg}$ ), with each slice acquired per breath-hold. Inversion times were adjusted to null normal myocardium (typically 320-440 msec, matrix size 256 $\times 256$ ). The LGE images were repeated in two separate phaseencoding directions to exclude artefacts. The scanning duration for each patient was around 45 minutes. Biventricular volumes and function were calculated using manual segmentation, using commercially available software (CMRtools; Cardiovascular Imaging Solutions, London, UK; or ADAS; Galgo Medical, Barcelona, Catalonia, Spain). Analyses of both the right and left ventricles were performed on a per slice basis by manual contouring of the endocardial and epicardial borders on the short-axis cine images, with contours manually drawn in enddiastole (start of R-wave) and in end-systole (smallest cavity area). Volumes were calculated based on the Simpson's method. The ventricular trabeculations and papillary muscles were excluded from the blood pool during manual segmentation. CMR imaging analysis was performed by two of five investigators, who were blinded to patient clinical status and eventual outcomes. In the event of disagreement, a third observer was asked to adjudicate.

The primary endpoint was a composite endpoint of heart failure hospitalisations, appropriate ICD shocks and cardiovascular mortality. Clinical outcomes were tracked by study members who did not participate in CMR imaging analysis, using a comprehensive electronic medical records system (CPSS2, iHIS, Singapore). Cardiovascular mortality was determined on 26 April 2016 via linkages with datasets of the National Registry of Diseases Office (NRDO), Singapore. The NRDO was established by the Ministry of Health, Singapore, to collect data on selected major diseases and health conditions, such as cancer, acute myocardial infarction, stroke and kidney failure, and to track the mortality status of all Singapore citizens and permanent residents. The study protocol conformed to the ethical guidelines of the 1975 Declaration of Helsinki, as reflected in a priori approval by the institution's human research committee (National Healthcare Group Domain Specific Review Board Approval number 2014/0424). Due to the use of a registered standing database and anonymised data, waiver of patient consent was granted by the ethics committee.

Continuous data and categorical variables were expressed as mean values \pm standard deviation and percentages, respectively. Continuous and categorical variables were assessed using unpaired $t$-tests and Fisher's exact tests, respectively. Pearson correlation was used to determine the relationship between two continuous variables. The log-rank test was used to compare Kaplan-Meier survival curves. To determine the association between clinical variables and the occurrence of primary endpoint, multivariate Cox proportional regression analyses were performed and results were expressed as hazard ratio (HR), with 95\% confidence interval $(\mathrm{Cl})$. A p-value $<0.05$ was considered to be statistically significant. All analyses were performed using IBM SPSS Statistics version 23.0 (IBM Corp, Armonk, NY, USA).

Mitral regurgitation was considered to be functional if anatomically normal mitral valve leaflets failed to coapt adequately due to global left ventricular remodelling secondary to DCM. The severity of functional mitral regurgitation was graded according to the American Society of Echocardiography guidelines. $^{(11)}$

\section{RESULTS}

A total of 86 consecutive patients, who fulfilled the inclusion criteria, were identified. Patients were predominantly male (80.2\%), with a mean echocardiographic LVEF of $30.1 \% \pm 12.7 \%$. LVEF, as determined by CMR imaging and echocardiography, was highly correlated $(r=0.78, p<0.01)$. Baseline characteristics of all patients as well as the subgroups with and without LGE enhancement are listed in Table I. 55 (64.0\%) patients had LGE on CMR imaging and constituted the LGE+ group. LGE was seen in the left ventricular mid-wall, epicardium, and superior and inferior right ventricular insertion points in $71.7 \%, 32.6 \%, 6.7 \%$ and $17.8 \%$ of patients, respectively (Fig. 1). The distribution of LGE is depicted in Fig. 2. According to the American Heart Association 17-segment model, the basal septal segments 2 and 3 were the most common segment to contain LGE. Within most segments, LGE was predominantly found in the mid-wall. Patients with LGE were much older $(p=0.002)$, had lower contractile function based on both echocardiography $(p=0.002)$ and CMR imaging ( $p<0.05)$, and were more likely to be receiving oral anticoagulants $(p=0.02)$. Mean follow-up was $4.9 \pm 3.2$ years. Thrombus was visible on CMR imaging in $4(4.7 \%)$ patients, of whom three had LGE.

In total, $23(26.7 \%)$ patients reached the composite endpoint, of which $19(34.5 \%)$ patients were in the LGE+ group and $4(12.9 \%)$ patients did not have LGE (LGE-; $p=0.01)$. Event-free survival at four years post CMR imaging was $96.8 \%$ in the LGEgroup when compared to $59.7 \%$ in the LGE+ group (Fig. 3). The difference between the two groups was driven largely by more appropriate ICD shocks and heart failure hospitalisations within the LGE+ group (Table II).

Increased age, higher New York Heart Association (NYHA) symptom status, anticoagulation therapy, lower echocardiographic LVEF and the presence of LGE were associated with increased likelihood of the occurrence of the primary endpoint (Table III). On multivariate logistic regression analysis, only increased age, use of anticoagulation (for stroke prevention in atrial arrhythmias) and presence of LGE remained statistically significant and independent risk markers for adverse clinical events.

\section{DISCUSSION}

In this consecutive series of Asian patients with DCM undergoing CMR imaging, the presence of LGE independently and strongly 
Table I. Patient demographics.

\begin{tabular}{|c|c|c|c|c|}
\hline \multirow[t]{2}{*}{ Variable } & \multicolumn{3}{|c|}{ No. (\%) } & \multirow[t]{2}{*}{ p-value* } \\
\hline & Total $(n=86)$ & LGE $+(n=55)$ & LGE- $(n=31)$ & \\
\hline $\operatorname{Age}^{\dagger}(y r)$ & $44.9 \pm 16.1$ & $48.7 \pm 14.2$ & $38.4 \pm 17.3$ & $<0.01^{\ddagger}$ \\
\hline Male gender & $69(80.2)$ & $43(78.2)$ & $26(83.9)$ & 0.52 \\
\hline Body mass index ${ }^{\dagger}\left(\mathrm{kg} / \mathrm{m}^{2}\right)$ & $26.0 \pm 5.5$ & $26.5 \pm 5.6$ & $25.0 \pm 5.1$ & 0.66 \\
\hline NYHA class & & & & 0.09 \\
\hline I-II & $73(84.9)$ & $44(80.0)$ & $29(93.5)$ & \\
\hline III-IV & $13(15.1)$ & $11(20.0)$ & $2(6.5)$ & \\
\hline QRS duration ${ }^{\dagger}$ (msec) & $106.2 \pm 26.9$ & $105.0 \pm 26.9$ & $109.0 \pm 27.4$ & 0.29 \\
\hline Echocardiographic LVEF ${ }^{\dagger}(\%)$ & $30.1 \pm 12.7$ & $27.2 \pm 12.5$ & $35.2 \pm 11.6$ & $<0.01^{\ddagger}$ \\
\hline \multicolumn{5}{|c|}{ Functional magnetic resonance imaging on echocardiography } \\
\hline Severe & $6(7.0)$ & $4(7.3)$ & $2(6.5)$ & 0.89 \\
\hline Moderate & $13(15.1)$ & $9(16.4)$ & $4(12.9)$ & 0.67 \\
\hline Mild & $24(27.9)$ & $20(36.4)$ & $4(12.9)$ & 0.20 \\
\hline Familial cardiomyopathy & $3(3.5)$ & $2(3.6)$ & $1(3.2)$ & 0.90 \\
\hline Diabetes mellitus & $22(25.6)$ & $16(29.1)$ & $6(19.4)$ & 0.32 \\
\hline Stroke & $9(10.5)$ & $6(10.9)$ & $3(9.7)$ & 0.86 \\
\hline Chronic lung disease & $14(16.3)$ & $10(18.2)$ & $4(12.9)$ & 0.52 \\
\hline Hyperlipidaemia & $36(41.9)$ & $26(47.3)$ & $10(32.3)$ & 0.18 \\
\hline Hypertension & $31(36.0)$ & $22(40.0)$ & $9(29.0)$ & 0.31 \\
\hline Atrial fibrillation/flutter & $8(9.3)$ & $6(10.9)$ & $2(6.5)$ & 0.49 \\
\hline ICD implanted during follow-up & $16(18.6)$ & $12(21.8)$ & $4(12.9)$ & 0.39 \\
\hline \multicolumn{5}{|l|}{ Medications } \\
\hline ACE inhibitor/ARB & $69(80.2)$ & $47(85.5)$ & $22(71.0)$ & 0.11 \\
\hline Beta-blocker & $62(72.1)$ & $43(78.2)$ & $19(61.3)$ & 0.09 \\
\hline Spironolactone & $31(36.0)$ & $24(43.6)$ & $7(22.6)$ & 0.06 \\
\hline Oral anticoagulant & $9(10.5)$ & $9(16.4)$ & $0(0)$ & $0.02^{\ddagger}$ \\
\hline Amiodarone & $11(12.8)$ & $6(10.9)$ & $5(16.1)$ & 0.49 \\
\hline \multicolumn{5}{|l|}{ CMR imaging parameter ${ }^{\dagger}$} \\
\hline Absolute LV EDV (mL) & $200.8 \pm 67.9$ & $204.8 \pm 67.0$ & $193.0 \pm 70.2$ & 0.23 \\
\hline $\operatorname{LVEF}(\%)$ & $36.9 \pm 12.2$ & $34.5 \pm 12.5$ & $41.6 \pm 10.2$ & $<0.01^{\ddagger}$ \\
\hline RVEF (\%) & $42.6 \pm 13.1$ & $40.4 \pm 12.9$ & $46.9 \pm 12.6$ & $0.02^{\ddagger}$ \\
\hline LV mass $(\mathrm{g})$ & $176.1 \pm 58.5$ & $177.5 \pm 62.0$ & $172.8 \pm 50.5$ & 0.38 \\
\hline Duration of follow-up ${ }^{\dagger}(y r)$ & $4.9 \pm 3.2$ & $4.1 \pm 3.0$ & $6.2 \pm 3.1$ & $<0.01^{\ddagger}$ \\
\hline
\end{tabular}

*p-value for comparison between the LGE + and LGE- groups. + Data presented as mean \pm standard deviation. $\neq \mathrm{p}<0.05$ was statistically significant. ACE: angiotensinconverting enzyme; ARB: angiotensin receptor blocker; CMR: cardiovascular magnetic resonance; EDV: end diastolic volume; ICD: implantable cardioverter-defibrillator; LGE: late gadolinium enhancement; LV: left ventricular; LVEF: left ventricular ejection fraction; MR: mitral regurgitation; NYHA: New York Heart Association; RVEF: right ventricular ejection fraction
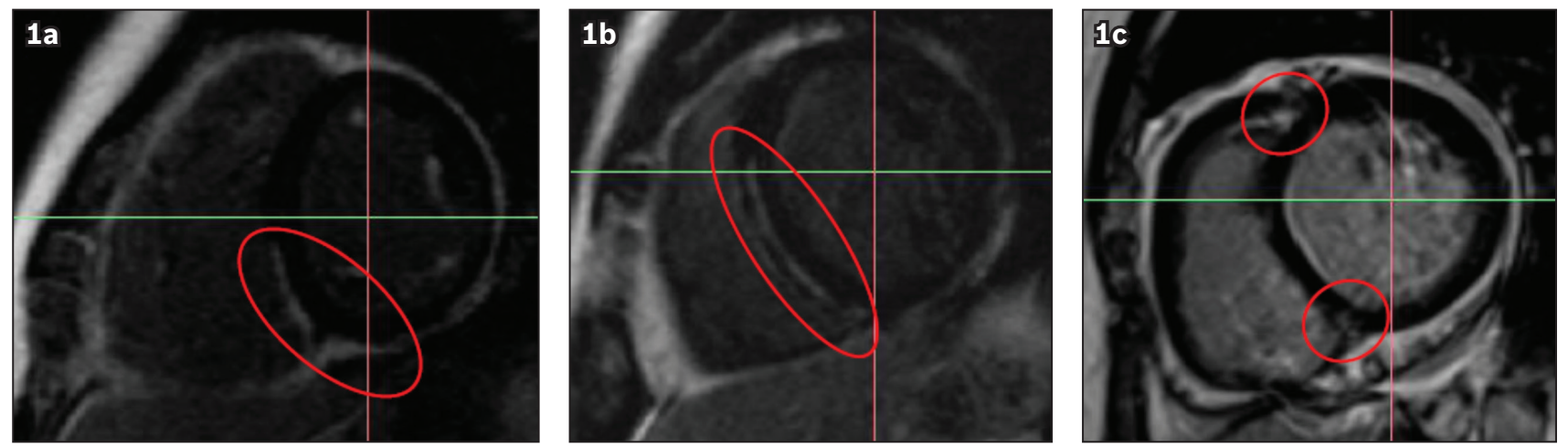

Fig. 1 Patterns of late gadolinium enhancement on cardiac magnetic resonance imaging of dilated cardiomyopathy show (a) epicardial scar, (b) mid-wall scar and (c) focal scarring. 


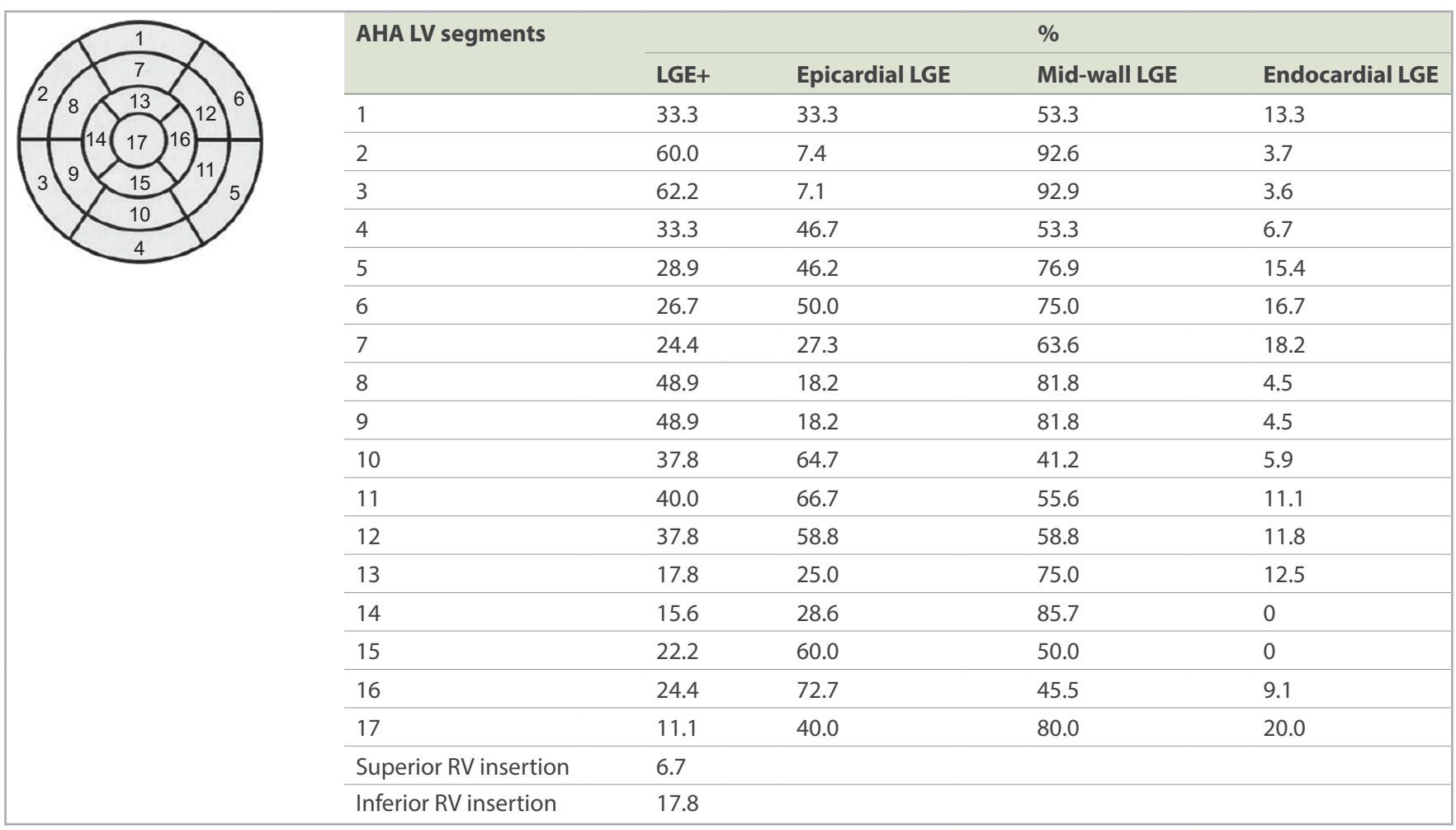

Fig. 2 Distribution of LGE based on AHA 17-segment model. AHA: American Heart Association; LGE: late gadolinium enhancement; LV: left ventricular; $\mathrm{RV}$ : right ventricular

Table II. Clinical endpoints.

\begin{tabular}{|llllll|}
\hline Variable & \multicolumn{3}{c}{ No. $(\%)$} & Hazard ratio $(\mathbf{9 5} \%$ Cl) & p-value \\
\cline { 2 - 4 } & Total $(\mathbf{n}=\mathbf{8 6})$ & LGE+ $(\mathbf{n}=\mathbf{5 5})$ & LGE- $(\mathbf{n}=\mathbf{3 1})$ & & \\
\hline Composite primary endpoint & $23(26.7)$ & $19(34.5)$ & $4(12.9)$ & $3.7(1.6-8.4)$ & 0.01 \\
\hline Heart failure hospitalisation & $9(10.5)$ & $7(12.7)$ & $2(6.5)$ & $2.4(0.6-9.2)$ & 0.19 \\
\hline Cardiovascular death & $12(14.0)$ & $8(14.5)$ & $4(12.9)$ & $1.4(0.4-4.4)$ & 0.46 \\
\hline Appropriate ICD shock & $7(8.1)$ & $6(10.9)$ & $1(3.2)$ & $4.7(0.8-16.0)$ & 0.11 \\
\hline
\end{tabular}

Cl: confidence interval; ICD: implantable cardioverter-defibrillator; LGE: late gadolinium enhancement

Table III. Predictors of primary composite endpoint.

\begin{tabular}{|c|c|c|c|c|}
\hline \multirow[t]{2}{*}{ Variable } & \multicolumn{2}{|c|}{ Univariate logistic regression analysis } & \multicolumn{2}{|c|}{ Multivariate logistic regression analysis } \\
\hline & Hazard ratio $(95 \% \mathrm{Cl})$ & p-value & Hazard ratio $(95 \% \mathrm{Cl})$ & p-value \\
\hline Age & $1.04(1.02-1.07)$ & $<0.01$ & $1.04(1.01-1.07)$ & 0.02 \\
\hline Echocardiographic LVEF & $0.95(0.91-0.98)$ & $<0.01$ & & \\
\hline NYHA class & $1.41(0.87-2.30)$ & 0.17 & & \\
\hline Anticoagulation & $2.61(1.11-6.17)$ & 0.03 & $2.61(1.09-6.23)$ & 0.03 \\
\hline LGE+ & $4.60(1.52-13.9)$ & $<0.01$ & $4.15(1.28-13.50)$ & 0.02 \\
\hline
\end{tabular}

Cl: confidence interval; LGE: late gadolinium enhancement; LVEF: left ventricular ejection fraction; NYHA: New York Heart Association

predicted an increased risk of heart failure hospitalisation, appropriate ICD shocks and cardiovascular mortality.

While mid-wall fibrosis is the hallmark and most common pattern of LGE distribution in DCM, other distribution patterns include subendocardial scarring extending towards the epicardium and patchy foci, particularly around right ventricular and left ventricular septal insertion points. ${ }^{(12)}$ All three patterns were seen in our cohort, with mid-wall fibrosis affecting $71.7 \%$ of patients.
On postmortem analysis, these sites of LGE corresponded to macroscopic replacement fibrosis. ${ }^{(7)}$ In a prospective longitudinal study of 472 patients with DCM undergoing CMR imaging, the presence and extent of mid-wall fibrosis was associated with a nearly threefold increased risk of all-cause mortality (annual mortality rates of $5.1 \%$ in patients with LGE when compared to $2.0 \%$ in patients without LGE) independent of LVEF and other conventional prognostic factors. ${ }^{(8)}$ The predictive ability of LGE to 


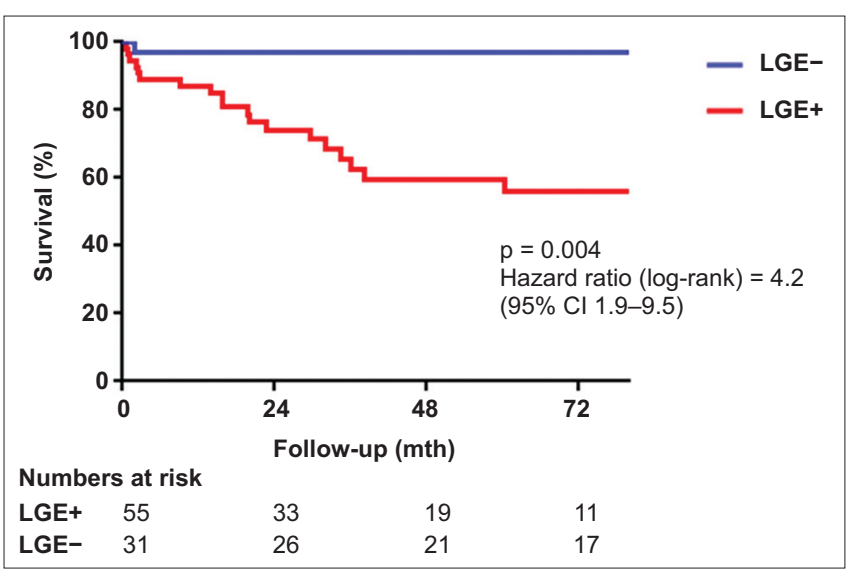

Fig. 3 Kaplan-Meier survival curves for primary composite endpoint. $\mathrm{Cl}$ : confidence interval; LGE: late gadolinium enhancement

predict adverse arrhythmic events was even stronger. In the same cohort, the presence of LGE was associated with a 5.2-fold increase in the likelihood of sudden death. These findings were further affirmed in the largest reported meta-analysis of 2,948 patients with DCM undergoing CMR imaging ( $44 \%$ of patients with LGE), whereby presence of LGE was associated with a relative 4.2 -fold increase in composite adverse arrhythmic endpoints consisting of sudden cardiac death, aborted cardiac arrest, sustained ventricular arrhythmias and appropriate ICD shocks. ${ }^{(1)}$ Interestingly, this association remained robust regardless of whether patients had severe or milder left ventricular dysfunction. In the subset of patients with primary prevention ICDs, the odds ratio of adverse arrhythmic events increased to 7.8 fold in patients with LGE compared to those without. The absence of LGE in patients with DCM was found to be associated with improved response to pharmacological and cardiac resynchronisation therapies. ${ }^{(13,14)}$

The demography of heart failure is significantly different between Caucasian and Asian cohorts, with the latter being younger but with higher rates of hypertension and diabetes mellitus. ${ }^{(15)}$ While the prognostic value of detecting LGE on CMR imaging was established for Caucasian cohorts and meta-analyses performed, Asian case series from Japan and China, and now ours from Singapore, reaffirm the identification of LGE as a powerful tool for prognostication of Asian patients with DCM. ${ }^{(13,16,17)}$ It is difficult to make further comparisons between Caucasian and Asian patient series, as there is significant heterogeneity in the profiles of patients reported in each series, inclusion criteria, choice of outcome measures and duration of follow-up. (1) Consequently, the proportion of patients with LGE on CMR imaging varies between $12 \%$ and $70 \%$.

ICD implantation is indicated for patients with DCM who have LVEF $\leq 35 \%$, and NYHA class II or III symptoms. ${ }^{(2,3)}$ These recommendations were largely based on the positive results of the SCD-HeFT study, which demonstrated life-saving benefits of ICD therapy in a mixed patient population with ischaemic and non-ischaemic cardiomyopathy, with mild-to-moderate heart failure symptoms. ${ }^{(18)}$ It has been well recognised from community cardiac arrest registries that $70 \%$ of patients with sudden death and premortem echocardiographic studies had LVEF that does not fulfil criteria for ICD. ${ }^{(19)}$
To date, to the best of our knowledge, no single randomised controlled trial of ICD therapy in patients with DCM has demonstrated statistically significant reduction in overall mortality, although significant reduction in arrhythmic mortality was seen in the DEFINITE and the recently completed DANISH studies. ${ }^{(20-22)}$ However, in an updated meta-analysis of primary prevention studies that included DANISH, ICD implantation was still associated with a $23 \%$ reduction of all-cause mortality. ${ }^{(23)}$ This was in contrast to the more convincing all-cause mortality reduction seen in primary prevention ICD trials that enrolled patients with ischaemic cardiomyopathy. ${ }^{(24)}$ The reasons for this distinction include DCM having lower absolute mortality rates, better response to pharmacological and cardiac resynchronisation therapies, and, importantly, poor specificity of echocardiographic LVEF criteria to differentiate among patients with DCM who are most likely to benefit from ICD therapy. A prospective randomised study incorporating CMR imaging findings into the decisionmaking process for primary prevention ICD implantation in fully optimised patients with DCM is strongly warranted, with the aim to develop a more sensitive approach to selecting patients with DCM for ICD implantation. In the meantime, clinicians should be cognisant that the presence of LGE on CMR imaging, which is not incorporated into current guidelines, represents a significant risk marker for adverse clinical events, including arrhythmia and sudden death. An individualised risk-benefit assessment of ICD implantation should be considered for patients with DCM having LGE who do not fulfil current guideline criteria.

This study was not without limitations. The relatively small sample size limited the precision of point estimates of individual adverse clinical outcomes. The decision to refer patients with DCM for CMR imaging was left to physician discretion and our cohort was therefore subject to referral bias. There were no postmortem comparisons made to CMR imaging scans.

In conclusion, the presence of LGE on CMR imaging was an independent predictor of adverse clinical events for Singapore patients with DCM. Routine use of CMR imaging to characterise the myocardial substrate is recommended for enhanced risk stratification and may guide clinical management.

\section{ACKNOWLEDGEMENTS}

This study was supported by an unrestricted research grant provided by Boston Scientific and Galgo Medical to the Department of Cardiology, National University Heart Centre Singapore, Singapore.

\section{REFERENCES}

1. Di Marco A, Anguera I, Schmitt M, et al. Late gadolinium enhancement and the risk for ventricular arrhythmias or sudden death in dilated cardiomyopathy: systematic review and meta-analysis. JACC Heart Fail 2017; 5:28-38.

2. Epstein AE, DiMarco JP, Ellenbogen KA, et al; American College of Cardiology Foundation; American Heart Association Task Force on Practice Guidelines; Heart Rhythm Society. 2012 ACCF/AHA/HRS focused update incorporated into the ACCF/AHA/HRS 2008 guidelines for device-based therapy of cardiac rhythm abnormalities: a report of the American College of Cardiology Foundation/ American Heart Association Task Force on Practice Guidelines and the Heart Rhythm Society. Circulation 2013; 127:e283-352.

3. McMurray JJ, Adamopoulos S, Anker SD, et al; ESC Committee for Practice Guidelines. ESC Guidelines for the diagnosis and treatment of acute and chronic 
heart failure 2012: The Task Force for the Diagnosis and Treatment of Acute and Chronic Heart Failure 2012 of the European Society of Cardiology. Developed in collaboration with the Heart Failure Association (HFA) of the ESC. Eur Hear J 2012; 33:1787-847.

4. Grimm W, Christ M, Bach J, Müller HH, Maisch B. Noninvasive arrhythmia risk stratification in idiopathic dilated cardiomyopathy: results of the Marburg Cardiomyopathy Study. Circulation 2003; 108:2883-91.

5. Andreu D, Ortiz-Pérez JT, Boussy T, et al. Usefulness of contrast-enhanced cardiac magnetic resonance in identifying the ventricular arrhythmia substrate and the approach needed for ablation. Eur Heart J 2014; 35:1316-26.

6. Nazarian S, Bluemke DA, Lardo AC, et al. Magnetic resonance assessment of the substrate for inducible ventricular tachycardia in nonischemic cardiomyopathy. Circulation 2005; 112:2821-5.

7. Assomull RG, Prasad SK, Lyne J, et al. Cardiovascular magnetic resonance, fibrosis, and prognosis in dilated cardiomyopathy. J Am Coll Cardiol 2006; 48:1977-85.

8. Gulati A, Jabbour A, Ismail TF, et al. Association of fibrosis with mortality and sudden cardiac death in patients with nonischemic dilated cardiomyopathy. JAMA 2013; 309:896-908.

9. Halliday BP, Gulati A, Ali A, et al. Association between midwall late gadolinium enhancement and sudden cardiac death in patients with dilated cardiomyopathy and mild and moderate left ventricular systolic dysfunction. Circulation 2017; 135:2106-15.

10. Lehrke S, Lossnitzer D, Schöb M, et al. Use of cardiovascular magnetic resonance for risk stratification in chronic heart failure: prognostic value of late gadolinium enhancement in patients with non-ischaemic dilated cardiomyopathy. Heart 2011; 97:727-32.

11. Zoghbi WA, Enriquez-Sarano M, Foster E, et al; American Society of Echocardiography. Recommendations for evaluation of the severity of native valvular regurgitation with two-dimensional and Doppler echocardiography. J Am Soc Echocardiogr 2003; 16:777-802.

12. Wu KC, Weiss RG, Thiemann DR, et al. Late gadolinium enhancement by cardiovascular magnetic resonance heralds an adverse prognosis in nonischemic cardiomyopathy. J Am Coll Cardiol 2008; 51:2414-21.

13. Ikeda $Y$, Inomata $T$, Fujita $T$, et al. Cardiac fibrosis detected by magnetic resonance imaging on predicting time course diversity of left ventricular reverse remodeling in patients with idiopathic dilated cardiomyopathy. Heart Vessels
$2016 ; 31: 1817-25$

14. Leyva F, Taylor RJ, Foley PW, et al. Left ventricular midwall fibrosis as a predictor of mortality and morbidity after cardiac resynchronization therapy in patients with nonischemic cardiomyopathy. J Am Coll Cardiol 2012; 60:1659-67.

15. Tromp J, Richards AM, Tay WT, et al. N-terminal pro-B-type natriuretic peptide and prognosis in Caucasian vs. Asian patients with heart failure. ESC Heart Fail 2018; 5:279-87.

16. Li X, Chan CP, Hua W, et al. Prognostic impact of late gadolinium enhancement by cardiac magnetic resonance imaging in patients with non-ischaemic dilated cardiomyopathy. Int J Cardiol 2013; 168:4979-80.

17. Liu T, Ma X, Liu W, et al. Late gadolinium enhancement amount as an independent risk factor for the incidence of adverse cardiovascular events in patients with stage C or D heart failure. Front Physiol 2016; 7:484.

18. Bardy GH, Lee KL, Mark DB, et al; Sudden Cardiac Death in Heart Failure Trial (SCD-HeFT) Investigators. Amiodarone or an implantable cardioverterdefibrillator for congestive heart failure. N Engl J Med 2005; 352:225-37.

19. Stecker EC, Vickers C, Waltz J, et al. Population-based analysis of sudden cardiac death with and without left ventricular systolic dysfunction: two-year findings from the Oregon Sudden Unexpected Death Study. J Am Coll Cardiol 2006; 47:1161-6.

20. Alba AC, Foroutan F, Duero Posada J, et al. Implantable cardiac defibrillator and mortality in non-ischaemic cardiomyopathy: an updated meta-analysis. Heart 2017; 104:230-6.

21. Køber L, Thune JJ, Nielsen JC, et al; DANISH Investigators. Defibrillator implantation in patients with nonischemic systolic heart failure. N Engl J Med 2016; 375:1221-30.

22. Kadish A, Dyer A, Daubert JP, et al; Defibrillators in Non-Ischemic Cardiomyopathy Treatment Evaluation (DEFINITE) Investigators. Prophylactic defibrillator implantation in patients with nonischemic dilated cardiomyopathy. N Engl J Med 2004; 350:2151-8.

23. Golwala H, Bajaj NS, Arora G, Arora P. Implantable cardioverter-defibrillator for nonischemic cardiomyopathy: an updated meta-analysis. Circulation 2017; 135:201-3.

24. Moss AJ, Zareba W, Hall WJ, et al; Multicenter Automatic Defibrillator Implantation Trial II Investigators. Prophylactic implantation of a defibrillator in patients with myocardial infarction and reduced ejection fraction. $\mathrm{N}$ Engl J Med 2002; 346:877-83. 